\title{
Aesthetics of assembly of giant molecules under precision synthesis
}

\author{
Gi Xue
}

The problems to efficiently and robustly pack the objects together have been studied for centuries due to their mathematic aesthetics and direct applications to understand the myriad phase structures compacted from spherical metallic atoms to other polyhedral building blocks. For the simplest polyhedron, rigid tetrahedra were shown to form crystalline and quasi-crystalline phases by analytical calculations or computer simulations if only packing or shape entropy dominates the free energy. However, related experimental observations of the structures or their formation mechanisms are remained largely unexplored, possibly mainly due to two reasons: firstly, enthalpy interaction in real system can not be ruled out as in modeling, and secondly, precise control of the shape and tuning of the enthalpy interaction of the building blocks are so far extremely challenging experimentally.

Recently, Prof. Stephen Z. D. Cheng from the University of Akron and his collaborators including Dr. Wen-Bin Zhang from Peking University have represented a rigorous work to show how to control the selective assembly of a class of nanosized giant tetrahedra by precisely mediating the interactions between the tetrahedra and the overall molecular geometry [1]. Various ordered supermolecular lattices, including the Frank-Kasper A15 phases, which are normally occurred in metal alloys are observed in this system. As shown in Fig. 1, the giant tetrahedra are constructed by attaching shape-persistent polyhedral oligomeric silsesquioxane (POSS) molecular nanoparticles with different hydrophobic (isobutyl) or hydrophilic (hydroxyl or carboxylic) functional groups at the vertices of a rigid tetrahedral framework. If the giant tetrahedron $\mathbf{1}$ contains four identical hydrophobic POSS cages, a triclinic unit cell crystalline structure with packing scheme to maximize the contacts among crystalline POSS cages is determined. Replacing one hydrophobic cage with a hydrophilic cage re- sults in giant tetrahedra 2a to 2c. As shown in Fig. 1b, such giant tetrahedra will form a density frustrated lamellar supramolecular structure dominated by the crystallization of hydrophobic cages. But after annealing the quenched melts, the Frank-Kasper A15 lattices with different periodicity were identified. The formation mechanism is very interesting: the melt giant tetrahedra will firstly differentiate into two types of spheres contained different numbers of giant tetrahedra, creating two types different coordination environment in a single component system, in contrast to metal alloys with different types of atoms. Such spheres with different sizes and deformability will further pack into A15 lattices. Further changing the functional groups, body centered cubic (bcc), double gyroid, cylinder or lamellae assembles that are determined by the competition between packing entropy and hydrophilic interaction are also observed.

By synthesis with atomic precision, Cheng and his collaborators have been pioneering in recent years the idea of molecular nanoparticles or sometimes called giant molecules précised derived from shape persistent POSS, fullerenes, polyoxometalates, proteins, and so on. Such system with tunable core structure and functionality has great potentials to building supermolecular lattices with larger length scale than from only atomic system. This new work is exciting. It is the first time in such detail to demonstrate the power of molecular symmetry with balanced enthalpy and entropy in creating thermodynamically stable supramolecular lattices.

Received 21 December 2015; accepted 22 December 2015; published online 25 December 2015

1 Huang MJ, Hsu CH, Wang J, et al. Selective assemblies of giant tetrahedra via precisely controlled positional interactions. Science, 2015, 348: 424-428

Department of Polymer Science and Engineering, Nanjing National Laboratory of Solid State Microstructures, Nanjing University, Nanjing 210093, China

Email:xuegi@nju.edu.cn 
a
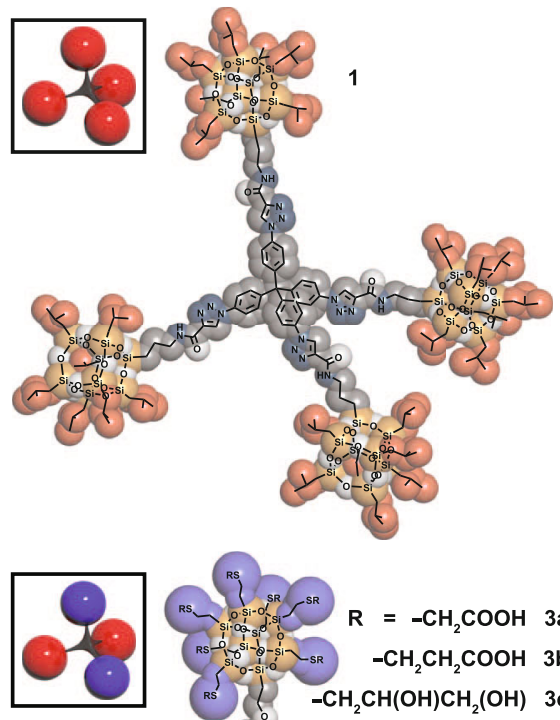

$R=-\mathrm{CH}_{2} \mathrm{COOH} 3 \mathrm{a}$ $-\mathrm{CH}_{2} \mathrm{CH}_{2} \mathrm{COOH} 3 \mathrm{~b}$ $-\mathrm{CH}_{2} \mathrm{CH}(\mathrm{OH}) \mathrm{CH}_{2}(\mathrm{OH}) 3 \mathrm{c}$

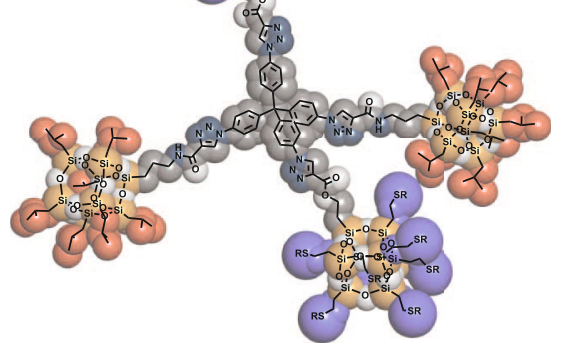

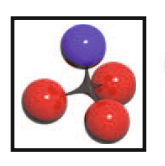

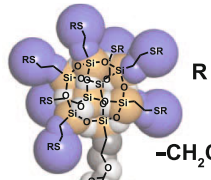

$\mathbf{R}=-\mathrm{CH}_{2} \mathrm{COOH} 2 \mathrm{a}$

$-\mathrm{CH}_{2} \mathrm{CH}_{2} \mathrm{COOH} 2 \mathrm{~b}$
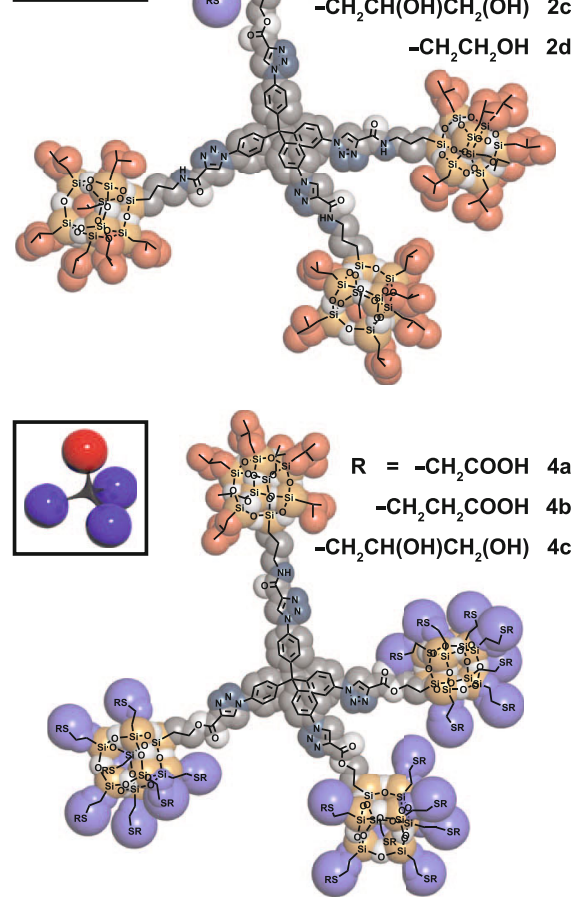

R: Hydrophilic functional group

b

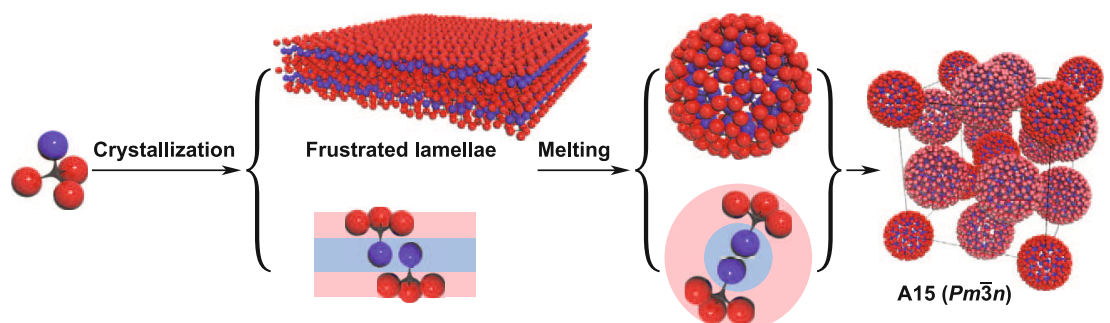

Figure 1 (a) The chemical structures and molecular models of the four categories of giant thetrahedra; (b) schematic illustrations of the selective assembly mechanism and molecular packing in the A15 lattice. Reprinted with permission from Ref. [1], Copyright 2015, The American Association for the Advancement of Sciences. 